\title{
Effect of Spatholobus suberectus (Fabaceae) extract on second-degree burns in rats
}

\author{
Lingyun Dai, Chunhu Zhao, Yu Jian, Zujun Mei, Xufeng Pei, Wensheng Yuan \\ and Fengxue $\mathrm{Wu}^{*}$ \\ The Second Clinical Medical College, Yangtze University, Jingzhou City, Hubei Province, 434020, China
}

*For correspondence: Email: fengxuewu134@hotmail.com; Tel/Fax: 0086-18107167468

Sent for review: 19 March 2017

Revised accepted: 16 September 2017

\begin{abstract}
Purpose: To evaluate the wound-healing effect of Spatholobus suberectus (Fabaceae) on seconddegree burns in a rat model.

Methods: The animals were divided into normal, negative control, as well as $10 \%$ Spatholobus suberectus (SS) (SS10), $20 \%$ SS (SS20) and standard (STD) groups. Second-degree burns were inflicted by exposing a $3 \times 3 \mathrm{~cm}$ sterile area of skin to boiling water for $10 \mathrm{~min}$. The animals were treated topically twice daily for 2 weeks. Wound contraction (\%) was measured after 2 weeks, while wound tissue histopathology was assessed by hematoxylin \& eosin and Masson's trichrome staining. In addition, lipid peroxidation (malondialdehyde kit) and cytokine secretion (ELISA) were measured in liver and plasma, respectively.

Results: The results of this study suggest that topical application of SS for 2 weeks significantly increases wound closure compared with the negative control. Moreover, treatment with SS significantly improved the pathological status of the wound throughout the protocol. There was also a significant decrease in malondialdehyde activity and increase in cytokine release in SS-treated rats compared with control rats.

Conclusions: The results show that topical application of SS after inflicting second-degree burns in rats results in increased wound healing and decreased cytokine release and oxidative stress.
\end{abstract}

Keyword: Spatholobus suberectus, Burns, Wound, Lipid peroxidation, Cytokines

Tropical Journal of Pharmaceutical Research is indexed by Science Citation Index (SciSearch), Scopus, International Pharmaceutical Abstract, Chemical Abstracts, Embase, Index Copernicus, EBSCO, African Index Medicus, JournalSeek, Journal Citation Reports/Science Edition, Directory of Open Access Journals (DOAJ), African Journal Online, Bioline International, Open-J-Gate and Pharmacy Abstracts

\section{INTRODUCTION}

Burn injuries are commonly encountered in primary and emergency care settings worldwide [1]. Approximately $1.5 \%$ of the global population experiences a burn each year, and many suffer from long-lasting effects such as scarring. Friction, chemicals and electricity can all result in burns and in the development of various complex symptoms via immune response activation. Previous studies suggest that infection control, pulmonary care, wound care and nutrition help improve recovery after a burn [2-4]. Currently, povidone iodine, mafenide acetate and silver sulfadiazine creams and ointments are available topically to improve wound healing after a burn [5-7]. However, there are still challenges to treating and healing burn wounds.

Several previous reports have shown that certain herbal extracts have the potential to improve healing after a burn [8-10]. Spatholobus suberectus (SS), which is found in China and belongs to the Leguminosae family, has been traditionally used for the treatment of several disorders, including rheumatism, anaemia and 
menstruation abnormalities [11]. Several reports have shown that SS has strong anti-platelet, antioxidant, neuroprotective and antiinflammatory abilities [12-13]. In addition, various compounds, including butin, liquiritigenin, eriodictyol and dihydrokaempferol, have been isolated from SS whole stems [14]. The aim of this study was to evaluate the effect of SS on wound healing after a second-degree burn.

\section{EXPERIMENTAL}

\section{Preparation of plant extracts}

Spatholobus suberectus stem was procured from a local vendor in spring season and authenticated by a taxonomist, Prof Yuangrun Zheng of Shanxi University, China. A voucher specimen (no. SU/2016/AU-09) was kept in the herbarium of the same university for future reference. The stems were cut into small pieces and dried in the shade. The stems were then boiled in distilled water before filtering through $150 \mu \mathrm{m}$ filter paper. This filtered material was mixed at 10 and $20 \% \mathrm{v} / \mathrm{v}$ with the vehicle gel and used for topical application.

\section{Animals}

Sprague-Dawley rats weighing $200-250 \mathrm{~g}$ were used in this study. All animals were housed under controlled conditions following institutional guidelines. All experiments were approved by the animal ethics committee of the Second Clinical Medical College, China (no. IEC/SCMC/2016/02) and the given study followed the guidelines of Association for the Assessment and Accreditation of Laboratory Animal Care International (AAALAC) for experimentation and animal use [15].

\section{Induction of second-degree burns}

The rats were divided into five different groups: normal, negative control (no treatment), SS10 (treatment with $10 \%$ SS), SS20 (treatment with $20 \%$ SS) and standard (STD: treatment with silver sulfadiazine). Treatments were given twice daily over a 2-week experimental period. To inflict burns, all rats were anesthetized with 50$100 \mathrm{mg} / \mathrm{kg}$ ketamine hydrochloride and $10 \mathrm{mg} / \mathrm{kg}$ xylazine, respectively. The dorsal surface of each rat was then shaved and the exposed area cleaned with ethanol. A second-degree burn was inflicted by exposing a $3 \times 3 \mathrm{~cm}$ section of the sterilized area to boiling water at $100{ }^{\circ} \mathrm{C}$ for 10 min. Excess water was then removed by draining it, and the burn was covered with gauze for $20 \mathrm{~s}$. The burns were examined throughout the experiment, and wound contraction and epithelialization were determined using Image Pro v. 6 software.

\section{Histological examination}

The rats were sacrificed at 4,7 and 14 days after wound induction, and tissues were collected from the burn area for examination. Tissue samples were fixed in $10 \%$ of formaldehyde and later embedded in paraffin. The samples were then cut into sections using a microtome. Sections were stained with hematoxylin and eosin ( $H$ \& E) or Masson's trichrome stain to detect changes in collagen content. A trinaocular microscope (model DM2500, Leica, Germany) was used to capture images of the tissues.

\section{Determination of lipid peroxidation}

Liver tissues were isolated from the rats and homogenized in a buffer solution [using a homogenizer. The supernatant was collected after spinning the homogenate $2000 \times$ for 10 min,, and malondialdehyde (MDA) activity was estimated using a MDA activity index kit (Oxis Research, USA) and by measuring the absorbance of the supernatant at $586 \mathrm{~nm}$.

\section{Evaluation of cytokine release}

Plasma was separated from the collected blood. Plasma concentrations of interleukin-6 (IL-6) and tumor necrosis factor alpha (TNF- $\alpha$ ) were estimated using an ELISA kit (R\&D Systems, USA). The detection limits of TNF- $\alpha$ and IL- 6 were 5.0 and $20.0 \mathrm{pg} / \mathrm{mL}$, respectively.

\section{Statistical analysis}

All data are shown as mean \pm standard deviation (SD, $n=10$ ). All results were analysed by oneway ANOVA and Dunnett's post hoc test (Gradpad prism 6.1., CA, USA). $P<0.05$ was considered statistically significant.

\section{RESULTS}

\section{Effect of SS treatment on wound healing}

Wound measurement was performed on days 1 , 4, 7 and 14 after burn induction. Increased formation of a light red crust was seen in the SS groups compared with the negative control group (Figure 1A).

SS treatment increased the percentage of wound closure (Figure 1B). There was a significant $(p<$ 0.01 ) increase in wound closure on days $4,7,11$ and 14 after burn induction compared with negative control group. 


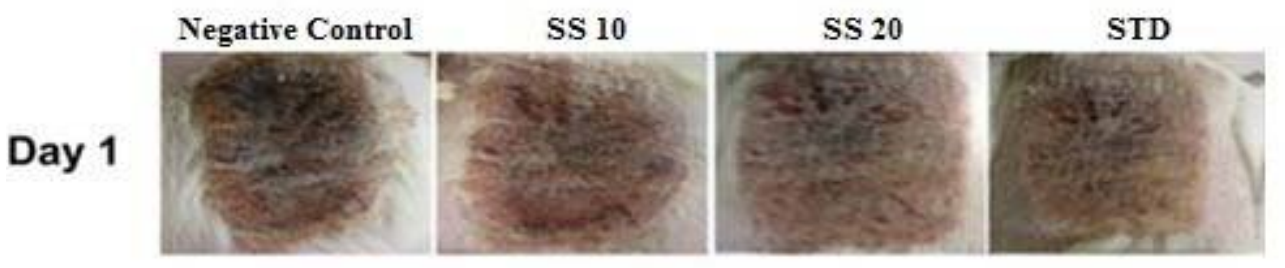

Day 4

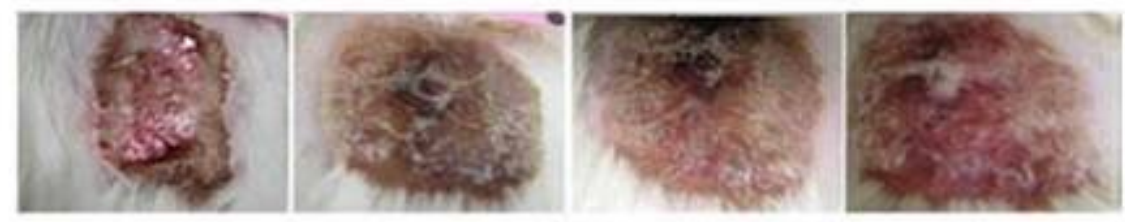

Day 7

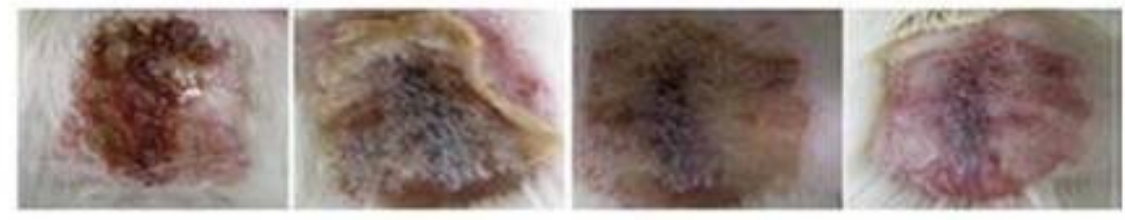

Day 14

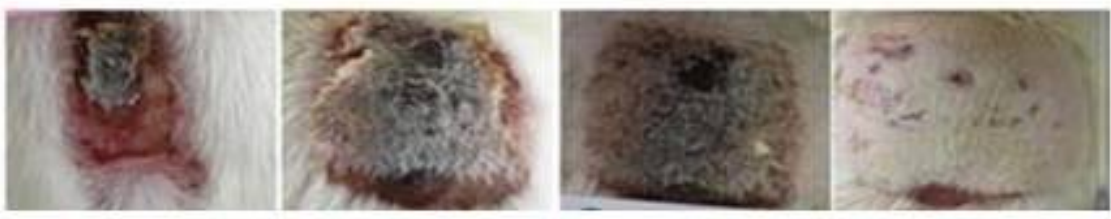

(A)

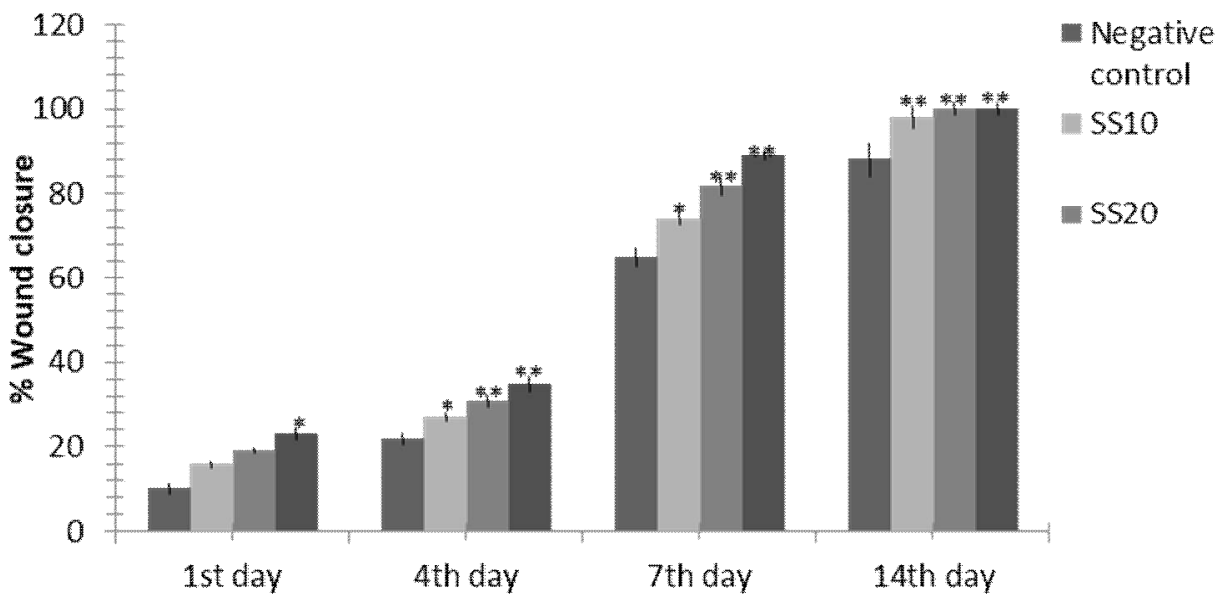

(B)

Figure 1: Effect of topical SS gel application on the healing of second-degree burns. A: photograph of the wounds; B: wound closure (\%); mean \pm SD $(n=10)$; \#\#p<0.01 vs normal group; ${ }^{* *} p<0.01$ vs negative control group

\section{Effect of SS on wound tissue histopathology}

In all groups, on day 4 after burn induction, histopathological examination of the wounds revealed deterioration of the dermal layer, phagocytic cell infiltration, absence of sebaceous glands, hair follicles and hyperaemic vessels, necrotic tissue remnants, scab formation and detachment of the epidermal layer. The SStreated groups showed no signs of congestion or oedema. On days 7 and 14 after burn induction, histopathological evaluation revealed increased angiogenesis, fibroblast infiltration and epithetlialization in the SS-treated groups compared with the negative control group (Table 1 and Figure 2).

Staining of the wound tissue with Masson's trichrome revealed clear morphological changes in the muscle fibres and levels of haemoglobin and keratin. These components stained red, as shown in the photos (Figure 3.). The pinkcoloured structures are adipose cells and cytoplasm, and the blue and brown structures 
are collagen fibres and nuclei, respectively. Low collagen content, scarring and clot formation are signs of wound inflammation, and all were observed in this study. Epithelial cell migration was also observed in the wounds, with increased epithelialization observed on day 7 after burn induction. Inflammatory cell content was significantly lower in the SS-treated groups compared with the negative control group. In addition, enhanced tissue healing with increased fibroblast proliferation, re-epithelialization and increased collagen content were observed in the SS-treated groups compared with the negative control group (Figure 3).

Table 1: Effect of SS on the histopathological score for burned tissue

\begin{tabular}{|c|c|c|c|c|c|c|c|c|c|c|c|c|}
\hline \multirow[t]{2}{*}{ Parameter } & \multicolumn{3}{|c|}{ Negative control } & \multicolumn{3}{|c|}{ SS10 } & \multicolumn{3}{|c|}{ SS20 } & \multicolumn{3}{|c|}{ STD } \\
\hline & Day 4 & Day 7 & Day 14 & Day 4 & Day 7 & Day 14 & Day 4 & Day 7 & Day 14 & Day 4 & Day 7 & Day 14 \\
\hline Oedema & + & + & - & - & - & - & - & - & - & - & - & - \\
\hline Congestion & ++ & + & - & + & - & - & - & - & - & - & - & - \\
\hline Necrosis & ++ & +++ & ++ & ++ & ++ & ++ & ++ & + & + & ++ & ++ & + \\
\hline proliferation & + & ++ & +++ & + & ++ & +++ & ++ & +++ & ++++ & ++ & ++++ & ++++ \\
\hline Angiogenesis & +++ & +++ & +++ & +++ & +++ & +++ & +++ & +++ & ++ & +++ & ++ & ++ \\
\hline Epithelialization & - & - & + & - & + & + & - & + & ++ & - & + & ++ \\
\hline Cell Infiltration & +++ & +++ & +++ & +++ & +++ & ++ & +++ & +++ & ++ & +++ & +++ & ++ \\
\hline
\end{tabular}

Slight: +; Moderate: ++; Marked: +++; Extensive: ++++; Absent: -
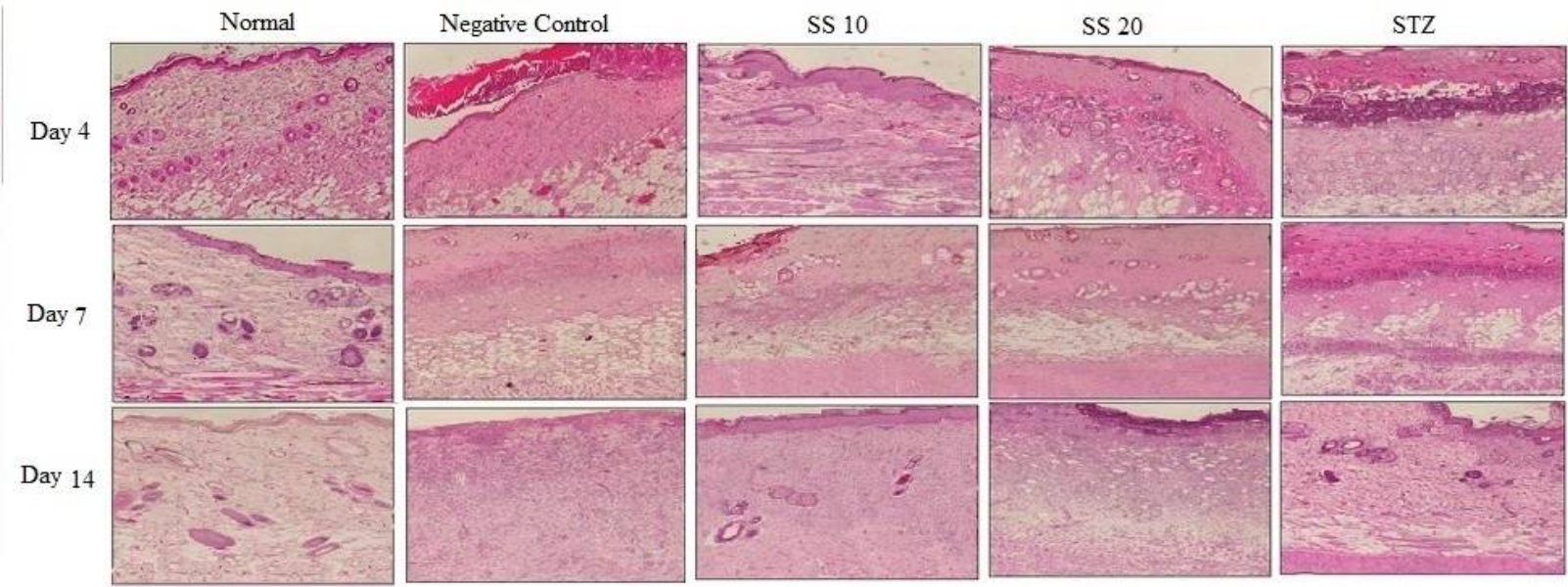

Figure 2: Effect of SS treatment on the histopathological characteristics of the burn skin using hematoxylin and eosin staining $(\mathrm{H} \& \mathrm{E} \times 4)$ on $1^{\mathrm{st}}, 4^{\text {th }}, 7^{\text {th }}$ and $14^{\text {th }}$ day of protocol. All photos are presented at $40 \times$ magnification

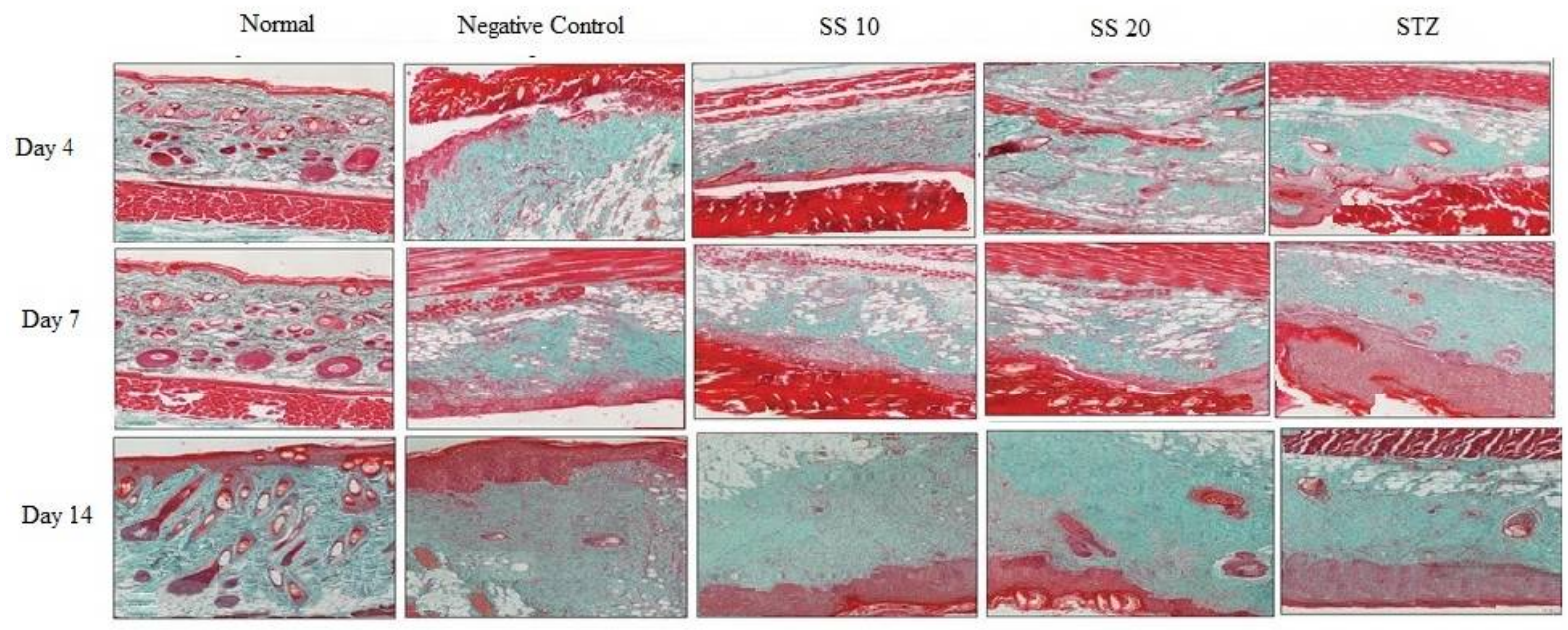

Figure 3: Effect of SS treatment on the histopathological characteristics of the burn skin using (Masson's trichrome staining, $\times 4$ ) on $1^{\text {st }}, 4^{\text {th }}, 7^{\text {th }}$ and $14^{\text {th }}$ day of protocol. All photos are presented at $40 \times$ magnification 


\section{Lipid peroxidation}

There was a significant increase $(p<0.01)$ in MDA level in the negative control group compared with the normal group. SS treatment significantly decreased $(p<0.01)$ MDA level compared with the negative control group (Figure 4).

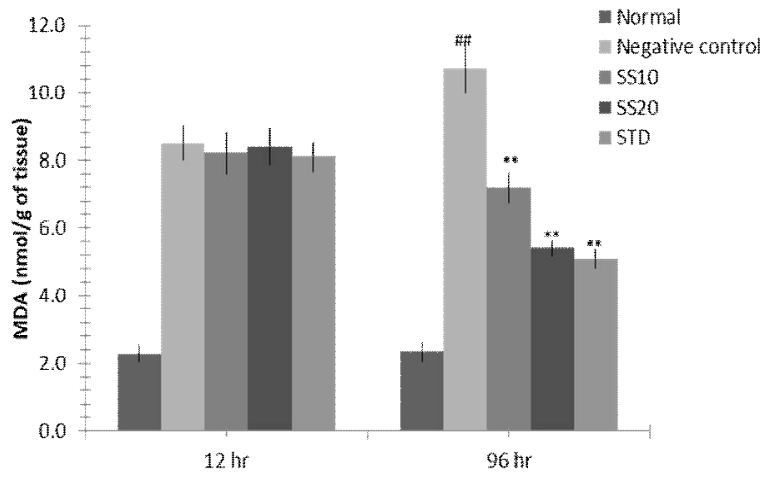

Figure 4: Effect of SS treatment on the level of MDA on $12^{\text {th }}$ and $96^{\text {th }} \mathrm{hr}$ after burn injury. Means \pm SD ( $\mathrm{n}=$ 10); \#\# $p<0.01$ vs. normal; ${ }^{* *} p<0.01$ vs. negative control
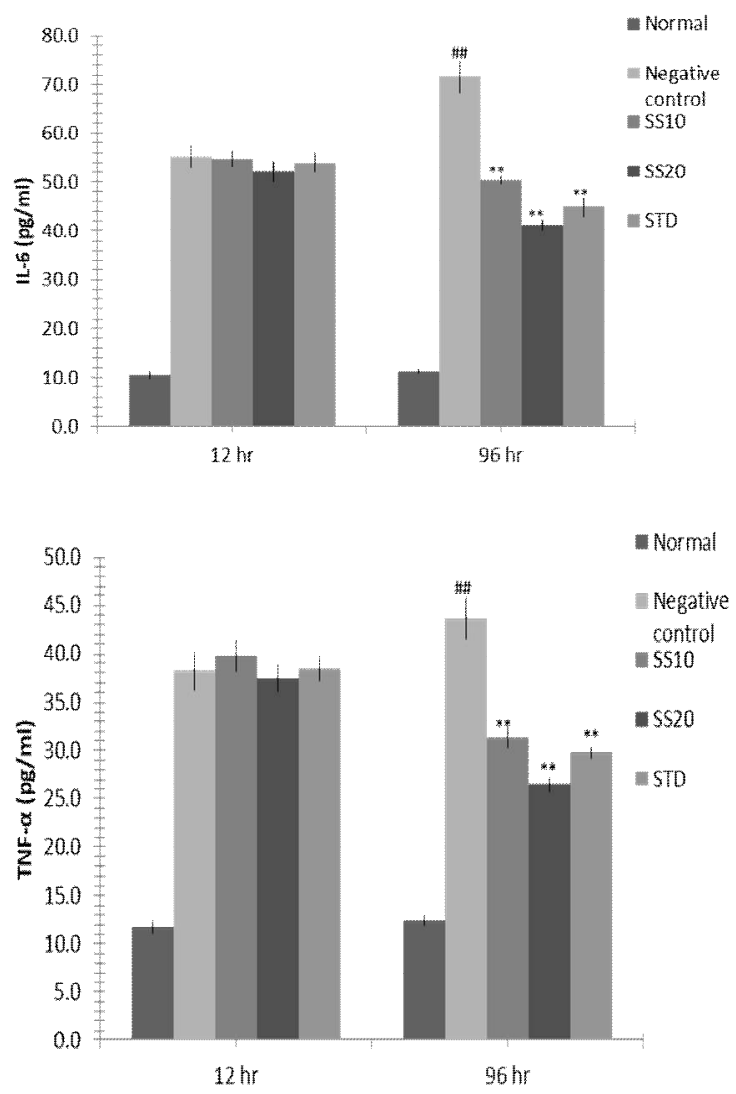

Figure 5: Effect of SS treatment on the secretion of IL- 6 and TNF- $\alpha$ in a burn-injury rat model on $12^{\text {th }}$ and $96^{\text {th }} \mathrm{hr}$ after burn injury. Mean $\pm \operatorname{SD}(\mathrm{n}=$ $10) ;{ }^{\# \#} p<0.01$ vs normal; ${ }^{\star \star} p<0.01$ vs negative control

\section{Cytokine secretion}

The effect of SS on cytokine secretion was estimated 12 and $96 \mathrm{~h}$ after wound induction (Figure 5). There were significant increases in the secretion of IL- 6 and TNF- $\alpha$ in plasma in all groups $12 \mathrm{~h}$ after wound induction compared with normal rats. However, rats treated with SS had significantly $(p<0.01)$ lower plasma cytokine levels $96 \mathrm{~h}$ after wound induction compared with the negative control group.

\section{DISCUSSION}

Burns are often encountered in primary and emergency care settings worldwide [1]. Although many treatments are available to manage burns, no available medication can cure a burn completely. Previous studies have shown that herbal medicines can potentially improve wound healing after a burn [8]. In this study, the effect of topical SS application on wound healing after a second-degree burn was evaluated. Wound healing was determined by evaluating histopathological changes using H\&E and Masson's trichrome staining 14 days after burn induction. Lipid peroxidation levels were also estimated by measuring MDA activity in the liver, and inflammation was estimated by measuring plasma cytokine levels (TNF- $\alpha$ and IL-6) 12 and $96 \mathrm{~h}$ after wound induction.

Previous studies have shown that lipid peroxidation results in inflammation after a burn injury via increased production of oxygen radicals [16]. Another study reported that lipid peroxidation causes cellular damage, necrosis, skin damage and increased capillary permeability [17]. Treatments that reduce lipid peroxidation by decreasing MDA activity may treat burn injuries effectively which supports the finding of this study.

In burn wound process of inflammation is started by WBCs, as they removes cell debris and microorganisms from the site of injury. Moreover it activates the production of inflammatory mediators such as TNF- $\alpha$ and IL- 6 and inflammation which enhances the vascular permeability, vasodilatation, fever and pain [18]. SS was found to inhibit the production of cytokines and thereby shows anti-inflammatory property. This anti-inflammatory property supports in the closure of wound with SS treatment.

Remodelling and repair of tissue requires collagens, matrix proteins and fibroblasts in the proliferative phase. Proliferation of fibroblast in the wound area produces several contents that 
are required for the repair of wound such as collagens, proteoglycan, fibronectin, hyaluronan and matrix proteins [19]. Data represented in the study suggested that treatment with SS enhances the fibroblast and collagen content in the wound tissue and thus reduces the period of epithelialisation.

\section{CONCLUSION}

The results of this study suggest that SS, applied topically, exerts strong wound healing activity on burned skin by reducing cytokine release and oxidative stress. There, therefore, a need for further investigation of SS for burns management.

\section{DECLARATIONS}

\section{Acknowledgement}

The authors would like to thank The Second Clinical Medical College, Yangtze University, China for providing the necessary facilities for this work.

\section{Conflict of Interest}

No conflict of interest associated with this work.

\section{Contribution of Authors}

The authors declare that this work was done by the authors named in this article and all liabilities pertaining to claims relating to the content of this article will be borne by them.

\section{Open Access}

This is an Open Access article that uses a funding model which does not charge readers or their institutions for access and distributed under the terms of the Creative Commons Attribution License (http://creativecommons.org/licenses/by/ 4.0) and the Budapest Open Access Initiative (http://www.budapestopenaccessinitiative.org/rea d), which permit unrestricted use, distribution, and reproduction in any medium, provided the original work is properly credited.

\section{REFERENCES}

1. Morgan ED, Bledsoe SC, Barker J. Ambulatory management of burns. Am. Fam. Physician 2000; 62: 2015-2026.

2. Zhang $Y M$, Fang $Y D$, Wang $Y C$, Wang SL, Lei ZY, Liu $X W$, Mao TC, Fan DL. Role of serum amyloid $P$ in skin graft survival and wound healing in burned patients receiving skin grafts. Clin. Chim. Acta 2011; 412: 227229.

3. Kahn SA, Beers RJ, Lentz CW. Use of acellular dermal replacement in reconstruction of non-healing lower extremity wounds. J. Burn Care Res. 2011; 32: 124-128.

4. Demirtas $Y$, Yagmur $C$, Soylemez F, Ozturk N, Demir A. Management of split-thickness skin graft donor site: $A$ prospective clinical trial for comparison of five different dressing materials. Burns 2010; 36: 999-1005.

5. Vloemans $A F$, Soesman $A M$, Suijker $M$, Kreis $R W$, Middelkoop E. A randomised clinical trial comparing a hydrocolloid-derived dressing and glycerol preserved allograft skin in the management of partial thickness. Burns 2003; 29: 702-710.

6. Hemmila MR, Mattar A, Taddonio MA, Arbabi $S$, Hamouda T, Ward PA, Wang SC, Baker JR. Jr. Topical nanoemulsion therapy reduces bacterial wound infection and inflammation after burn injury. Surgery 2010; 148: 499-509.

7. Steen M. Review of the use of povidone-iodine (PVP-I) in the treatment of burns. Postgrad. Med. J. 1993; 69: S84S92.

8. Kelly AP, Taylor SC. Dermatology for skin of color. China: McGraw-Hill; 2009. p. 42-3.

9. Krishnan $P$. The scientific study of herbal wound healing therapies: Current state of play. Curr Anaesth Crit Care 2006; 17: 21027.

10. Liu M, Dai Y, Li Y, Luo Y, Huang F, Gong Z, Meng Q. Madecassoside isolated from Centella asiatica herbs facilitates burn wound healing in mice. Planta Med 2008; 74: 809?815.

11. Lee $M H$, Lin YP, Hsu FL, Zhan GR, Yen KY. Bioactive constituents of Spatholobus suberectus in regulating tyrosinase-related proteins and mRNA in HEMn cells. Phytochemistry 2006; 67(12): 1262-1270.

12. Lam TL, Lam ML, Au TK, Ip DT, Ng TB, Fong WP, Wan DC. A comparison of human immunodeficiency virus type-1 protease inhibition activities by the aqueous and methanol extracts of Chinese medicinal herbs. Life Sciences 2000; 67: 2889-2896.

13. Li RW, David-Lin G, Myers SP, Leach DN. Antiinflammatory activity of Chinese medicinal vine plants. J Ethnopharmacol. 2003; 85: 61-67.

14. Yen KY. 1992. The illustrated Chinese materia medica. SMC Publishing Inc., Taipei.

15. Guide for the Care and Use of Laboratory Animals: Eighth Edition Committee for the Update of the Guide for the Care and Use of Laboratory Animals; National Research Council. 2010; ISBN: 0-309-15401-4.

16. Hiramatsu M, Izawa $Y$, Hagihara M, Nishigaki I, Yagi $K$. Serum lipid peroxide levels of patients suffering from thermal injury. Burns Incl. Therm. Inj. 1942; 11: 111-116.

17. Cetinkale $O$, Belce A, Konukoglu D, Senyuva $C$, Gumustas MK, Tas T. Evaluation of lipid peroxidation and total antioxidant status in plasma of rats following thermal injury. Burns 1997; 23: 114-116.

18. Schwacha MG. Macrophages and post-burn immune dysfunction. Burns 2003; 29: 1-14.

Trop J Pharm Res, October 2017; 16(10): 2370 
Dai et al

19. Teller $P$, White TK. The physiology of wound healing: Injury through maturation. Surg Clin North Am 2009; 89:

599-610. 